\title{
Los índices de pacientes adultos con sobrepeso u obe- sidad, asistidos por nutricionistas en el Hospital Mater- no Infantil, barrio Loma Pytá, en la ciudad de Asunción, Paraguay, 2019
}

Indexes of adult patients with overweight or obesity, assisted by nutritionists at the Maternal and Child Hospital, Loma Pytá neighborhood, in the city of Asunción, Paraguay, 2019

Everton Souza $^{1}$; Suzaneide Siqueira ${ }^{1}$; Mirta Dennis ${ }^{1}$

\section{RESUMEN}

La obesidad es una enfermedad caracterizada por la acumulación excesiva de grasa corporal, que produce efectos nocivos para la salud. El índice de masa corporal (IMC) es un indicador simple de la relación entre el peso y la talla que se utiliza frecuentemente para identificar el sobrepeso y la obesidad en los adultos. Se calcula dividiendo el peso de una persona en kilos por el cuadrado de su talla en metros ( $\mathrm{kg} / \mathrm{m} 2)$. Desde 1975, la obesidad se ha casi triplicado en todo el mundo. Actualmente, se ha considerado el trastorno nutricional más importante en los países desarrollados y en desarrollo, debido a su mayor incidencia. El objetivo del estudio fue determinar los niveles de sobrepeso $\mathrm{u}$ obesos en pacientes que consultaron nutricionistas en el Hospital Materno Infantil de Loma Pytá, de Asunción durante el mes de octubre de 2019. Fue un estudio no experimental, observacional descriptivo que incluyó 78 pacientes de ambos sexos diagnosticados con exceso de peso. El 73\% de los pacientes diagnosticados, correspondieron al sexo femenino, edad de $18 \pm 80$ años. La mayoría de los pacientes que buscaban ayuda médica nutricional ya eran en gran mayoría obesos, teniendo en vista el soma de los que tenían grado de obesidad I, II y III, equivaliendo a $62 \%$ de los pacientes y el $38 \%$ restante eran pacientes con sobrepeso diagnosticado. Treinta pacientes con sobrepeso (38\%), 27 pacientes con obesidad grado I (35\%), 12 pacientes con obesidad grado II (15\%) y 9 pacientes con obesidad grado III (12\%). En el grado de obesidad de cada género, poco más de $90 \%$ de los pacientes masculinos era entre hombres con sobrepeso y con obesidad grado I (leve). En los porcentuales femeninos, 16\% de ellas tienen obesidad grado III y $17 \%$ obesidad grado II.

Palabras clave: Sobrepeso; Obesidad; Enfermedades asociadas.

Fecha de recepción: junio 2020; fecha de aceptación: julio 2020

${ }^{1}$ Universidad Privada María Serrana, Carrera de Medicina. Asunción, Paraguay

Autor de correspondencia: Mirta Dennis. Email: mirtha_denis2909@hotmail.com 


\section{ABSTRACT}

Obesity is a disease characterized by excessive accumulation of body fat, which produces harmful health effects. The body mass index (BMI) is a simple indicator of the relationship between weight and height that is frequently used to identify overweight and obesity in adults. It is calculated by dividing a person's weight in kilos by the square of their height in meters $(\mathrm{kg} / \mathrm{m} 2)$. Since 1975, obesity has almost tripled worldwide. Currently, it has been considered the most important nutritional disorder in developed and developing countries, due to its higher incidence. The objective of the study was to determine the levels of overweight or obese in patients who consulted nutritionists at the Maternal and Child Hospital of Loma Pytá, Asunción during the month of October 2019 It was a non-experimental, observational descriptive study that included 78 patients of both sexes diagnosed overweight. $73 \%$ of the diagnosed patients were female, aged 18 \pm 80 years. Most of the patients seeking nutritional medical help were already largely obese, taking into account the soma of those with obesity grade I, II and III, equivalent to $62 \%$ of patients and the remaining $38 \%$ were patients with diagnosed overweight. Thirty overweight patients (38\%), 27 patients with grade I obesity (35\%), 12 patients with grade II obesity (15\%) and 9 patients with grade III obesity (12\%). In the degree of obesity of each gender, just over $90 \%$ of the male patients were among overweight men and with obesity grade I (mild). In female percentages, $16 \%$ of them have grade III obesity and $17 \%$ grade II obesity.

Keywords: Overweight; Obesity; Associated diseases. 


\section{INTRODUCCIÓN}

La obesidad es una enfermedad caracterizada por la acumulación excesiva de grasa corporal a un nivel que compromete la salud de las personas, causando daños como cambios metabólicos, trastornos respiratorios y locomotores. Además de ser un factor de riesgo para enfermedades cardiovasculares, diabetes mellitus tipo II y algunos tipos de cáncer (1).

El diagnóstico de obesidad se basa en el parámetro estipulado por la Organización Mundial de la Salud - OMS: Body Mass Index (BMI) o el Índice de Masa Corporal (IMC), obtenido de la relación entre el peso corporal $(\mathrm{kg})$ y la estatura $(\mathrm{m} 2)$ de individuos. Se calcula dividiendo el peso de una persona en kilos por el cuadrado de su talla en metros $(\mathrm{kg} / \mathrm{m} 2)$. A través de este parámetro, las personas cuyo IMC es igual o superior a $25 \mathrm{~kg} / \mathrm{m} 2$ tienen sobrepeso e igual o superior a $30 \mathrm{~kg} / \mathrm{m} 2$ son obesas (1).

\section{MÉTODO}

El estudio se ejecutó sobre la base de la búsqueda de informaciones a través de las historias clínicas de los pacientes con sobrepeso u obesos que consultaron nutricionistas en el Hospital Materno Infantil de Loma Pytá, de la ciudad de Asunción, en el mes de octubre de 2019. Fue un estudio no experimental, observacional descriptivo, retrospectivo. La población muestral estuvo compuesta de 78 pacientes, siendo 57 mujeres y 21 hombres con sobrepeso u obesidad diagnosticado y, si los hay, con su enfermedad asociada.

\section{Criterios de Inclusión:}

- Pacientes adultos con sobrepeso u obesos que acudieron a consulta con nutricionistas en el Hospital Infantil Materno del barrio Loma Pytá, en la ciudad de Asunción, en el mes de octubre de 2019.

- Ambos sexos

- Pacientes que poseían registrados sus
Según las proyecciones del gobierno, través de la Dirección General de Estadística, Encuestas y Censos, en 2019, el Paraguay tiene una población de 7.152 .703 habitantes, siendo en su mayoría de 64\%, compuesta por personas entre 15 a 64 años de edad (2). Los datos del Ministerio de la Salud y Bien Estar Social afirman que 23\% de los adultos padece obesidad, o sea, uno de cada cuatro adultos. Cuando se incluye las personas con sobrepeso, eses datos se elevan a $58 \%$, en donde dos de cada tres paraguayos poseen sobrepeso u obesidad (3).

Considerando estas estadísticas generales se planteó como objetivo del estudio determinar los niveles de sobrepeso u obesos en pacientes que consultaron nutricionistas en el Hospital Materno Infantil de Loma Pytá, de Asunción durante el mes de octubre de 2019.

datos completos referentes a edad, género, peso, talla, IMC calculado y diagnostico correspondiente en los archivos del Hospital supra citado.

\section{Criterios de Exclusión:}

- Pacientes con sobrepeso u obesos acudidos fuera del marco temporal preestablecido.

- Pacientes sin sobrepeso u obesidad.

- Pacientes no acudidos por nutricionistas.

- Pacientes menores de edad.

- Pacientes con obesidad decurrente de embarazo.

- Pacientes que no poseían registrados sus datos completos referentes a edad, género, peso, talla, IMC calculado y diagnostico correspondiente en los archivos del hospital supra citado.

\section{Aspectos Éticos}

Se elaboró una carta de solicitud de permiso para el Director General del Hospital 
Materno Infantil de Loma Pytá, para que permitiese el acceso a los históricos de los pacientes en el mes de octubre de 2019 y los datos estadísticos del hospital de este período; explicando también la finalidad exclusivamente académica y procedimiento de la investigación. No se revelarán los nombres de los pacientes incluidos en la investigación.

\section{RESULTADOS}

En el Gráfico 1 se muestra que el $73 \%$ de los pacientes eran del sexo femenino, en primer lugar, esto podría indicar que las

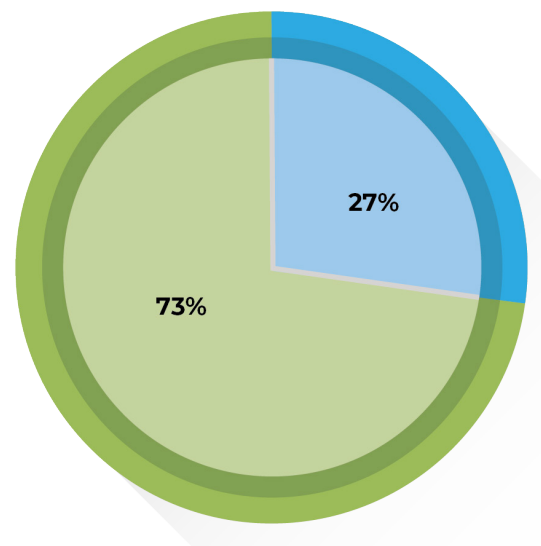

Gráfico 1. Distribución de pacientes por sexo.

El Gráfico 2, muestra la notable mayoría femenina que buscó ayuda médica por problemas de sobrepeso, se especifica género y rango de edad.

En ambos sexos, es importante resaltar el bajo interés en la búsqueda por asistencia médica nutricional por parte de adultos jóvenes de 18 a 40 años (solo 12 pacientes, $15 \%$ del total) y que, a partir de los 40 años, existe una creciente demanda de ayuda médica nutricional.

\section{Variables}

Se consideraron las siguientes variables: sexo, edad, grado de obesidad según IMC diagnosticado y enfermedades asociadas al exceso de peso diagnosticadas de cada paciente del cual se obtendrán las muestras para el estudio.

Los datos serán obtenidos fueron digitalizados y analizados en planilla Excel. mujeres buscaban ayuda médica más que los hombres pro el exceso de peso.
Hombres

Mujeres
Otro punto importante a considerar es la reducción repentina o interrupción de la demanda de monitoreo nutricional en los ancianos con exceso de peso. Este hecho pudiera indicar estas suposiciones: una que debido a las limitaciones de movilidad naturales por su edad y agravados por el grado de obesidad, se le dificulta acudir al nutricionista ya que pudieran depender de otra persona los lleve, y la otra que podría estar asociada al deceso. 


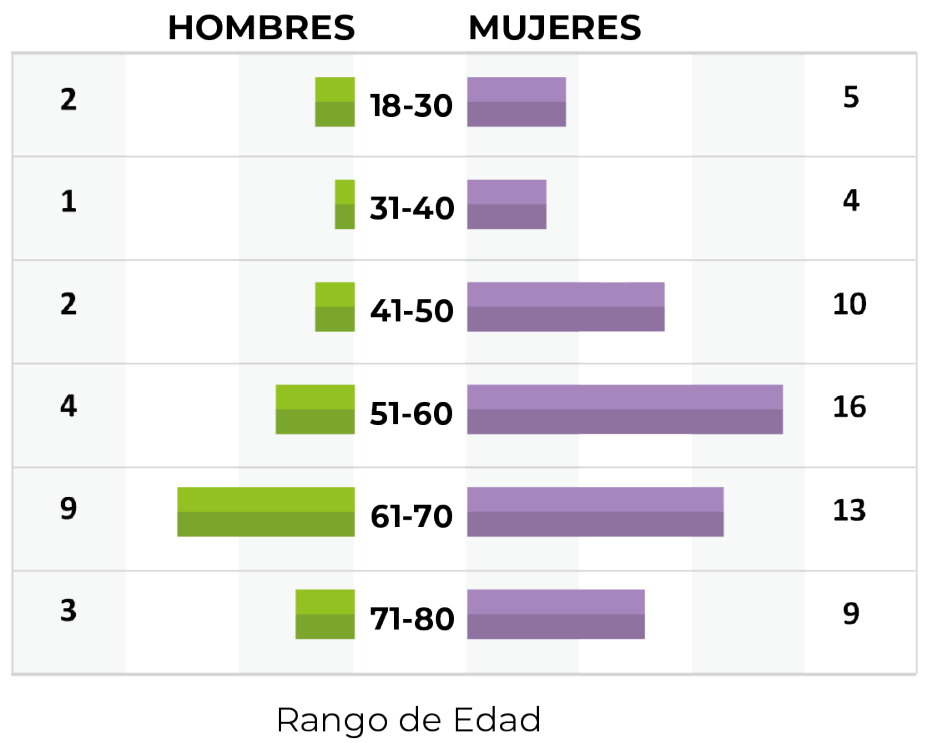

Gráfico 2. Cantidad de pacientes que acudieron a consulta distribuidos por edad y sexo.

Los datos referentes al grado de obesidad en que se encuentran los pacientes de ambos sexos, indican que la mayoría de los pacientes que buscan ayuda médica nutricional ya son en gran mayoría obesos, teniendo en vista el soma de los que tienen grado de obesidad I, II y III, equivaliendo a $62 \%$ de los pacientes y el $38 \%$ restante son pacientes con sobrepeso diagnosticado. (Ver Gráfico 3)
En el Gráfico 4 los resultados reflejan que de la muestra de 78 pacientes, de ambos sexos, 30 de ellos tienen sobrepeso (38\%), 27 pacientes con obesidad grado I (35\%), 12 pacientes con obesidad grado II (15\%) y 9 pacientes con obesidad grado III $(12 \%)$.

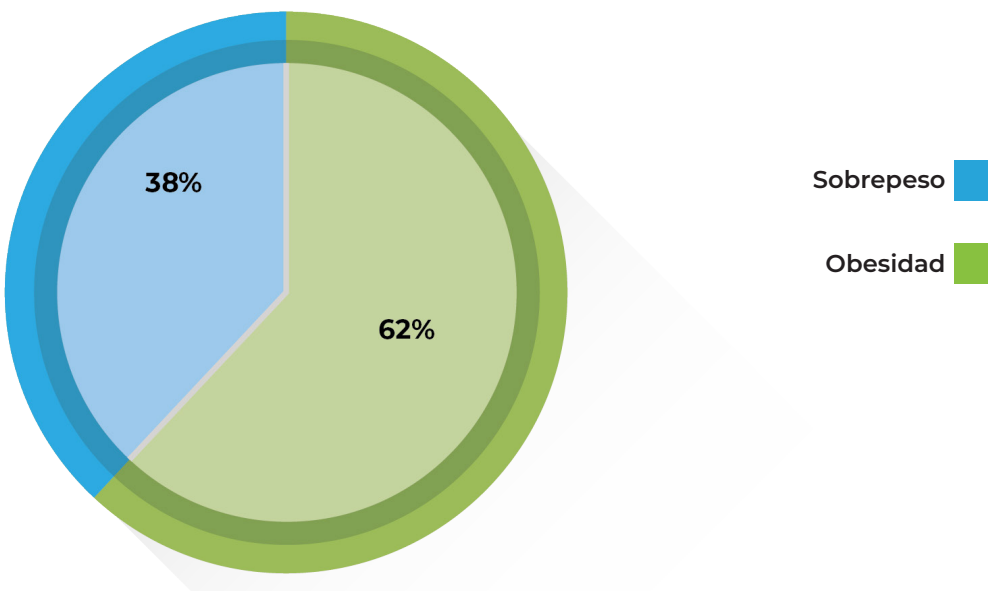

Gráfico 3. Clasificación de pacientes según sobrepeso y obesidad. 


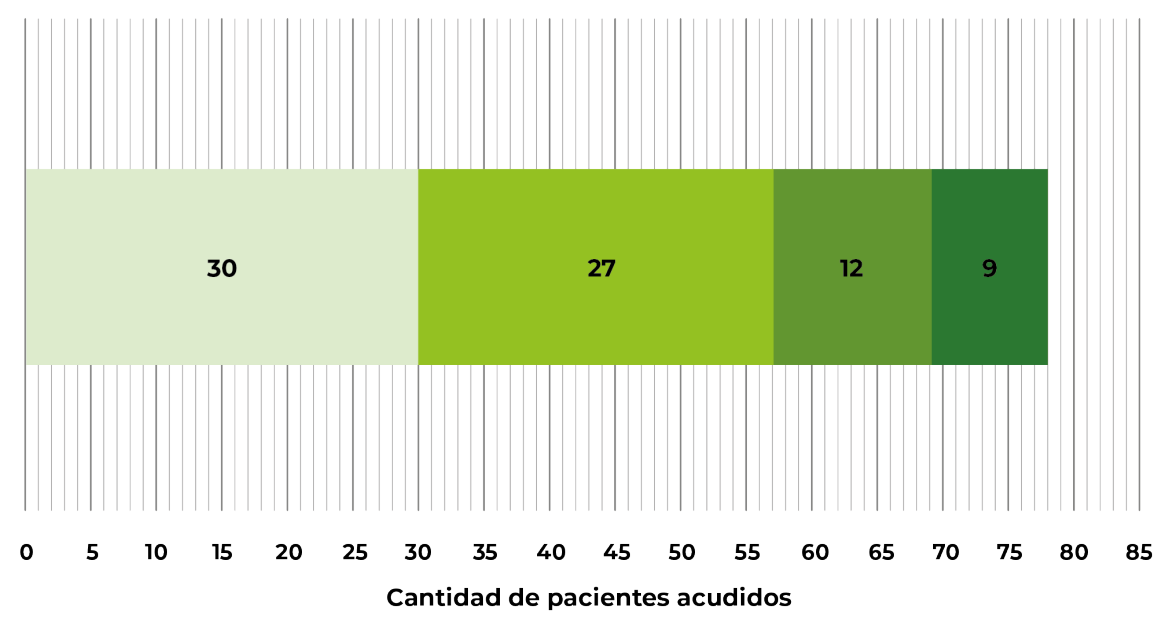

Sobrepeso Obesidad grado I Obesidad grado I Obesidad grado I

Gráfico 4. Grado de obesidad y sobrepeso de los pacientes de la muestra

El Gráfico 2, muestra la notable mayoría femenina que buscó ayuda médica por problemas de sobrepeso, se especifica género y rango de edad.

En ambos sexos, es importante resaltar el bajo interés en la búsqueda por asistencia médica nutricional por parte de adultos jóvenes de 18 a 40 años (solo 12 pacientes, $15 \%$ del total) y que, a partir de los 40 años, existe una creciente demanda de ayuda médica nutricional.

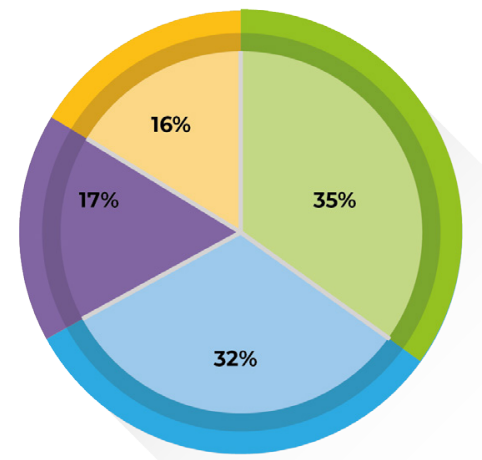

Gráfico 5. Grado de obesidad de pacientes mujeres.
Otro punto importante a considerar es la reducción repentina o interrupción de la demanda de monitoreo nutricional en los ancianos con exceso de peso. Este hecho pudiera indicar estas suposiciones: una que debido a las limitaciones de movilidad naturales por su edad y agravados por el grado de obesidad, se le dificulta acudir al nutricionista ya que pudieran depender de otra persona los lleve, y la otra que podría estar asociada al deceso. 


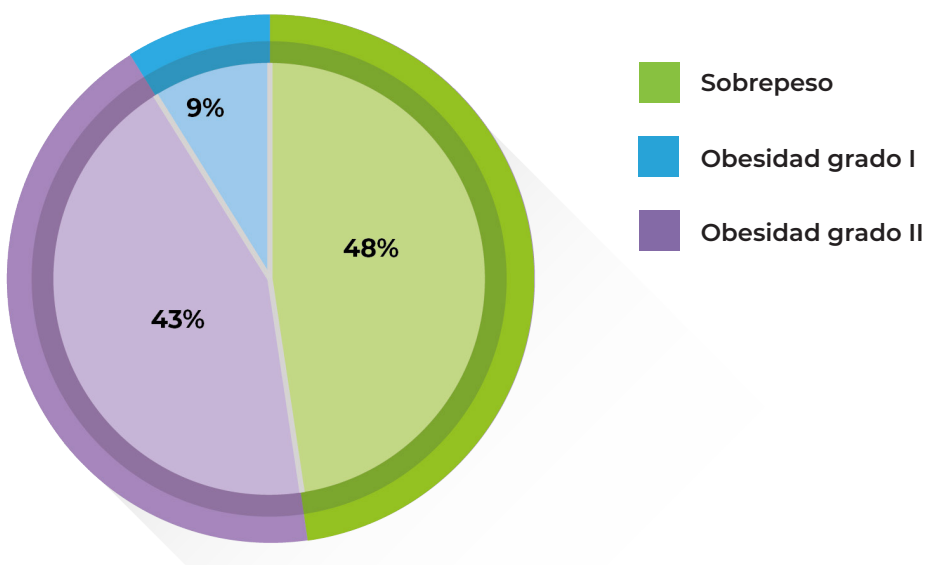

Gráfico 6. Grado de obesidad de pacientes hombres.

En los Gráficos 7 y 8 se especifican por te por grado de obesidad siendo 57 mujegénero, mujeres y hombres respectivamen- res y 21 hombres respectivamente.

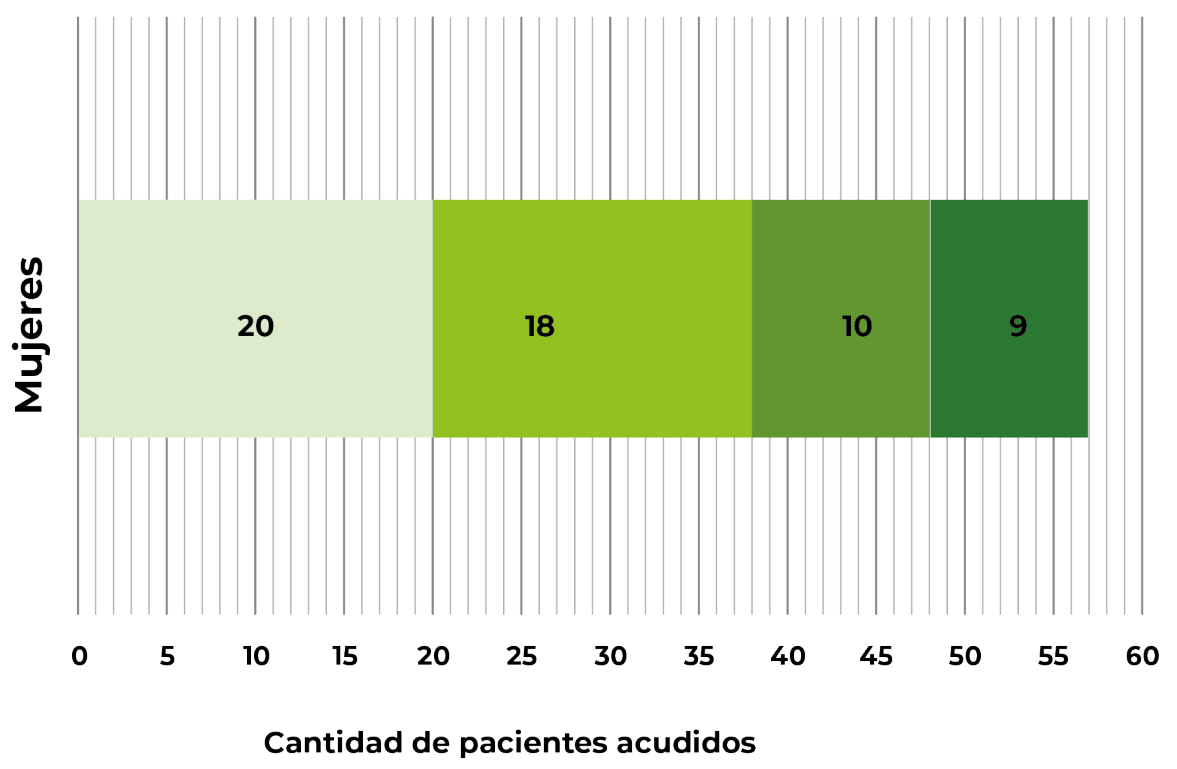

Sobrepeso Obesidad grado I Obesidad grado II Obesidad grado III

Gráfico 7. Grado de obesidad en pacientes mujeres. 


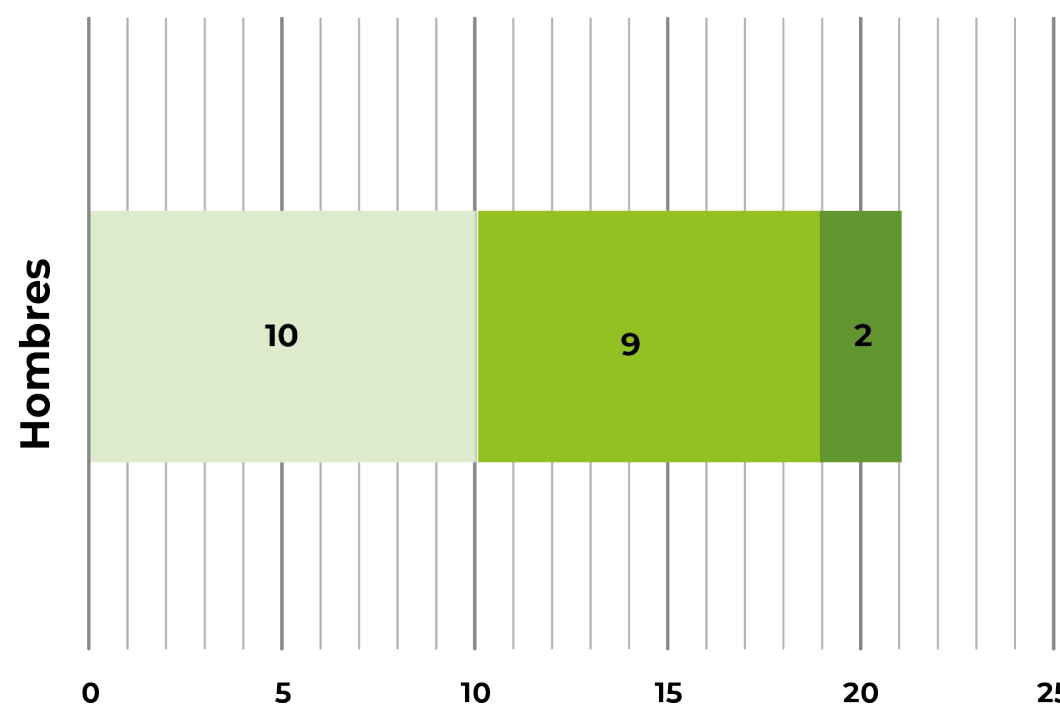

Cantidad de pacientes acudidos

\section{Sobrepeso Obesidad grado I Obesidad grado II}

Gráfico 8. Grado de obesidad en pacientes hombres.

Al observar el Gráfico 7, sobre el grado de obesidad de pacientes mujeres y dividirlos en 3 tercios distintos se determina que en el primer tercio se concentran 20 pacientes diagnosticadas con sobrepeso (35\%); en el segundo tercio 18 pacientes con obesidad grado I (32\%) y el tercer tercio 19 pacientes con grados de obesidad más elevados $(33 \%)$, equivalente a la suma de los porcentajes de obesidad grado II y III), discriminados en 10 pacientes con obesidad grado II (17\%) y 9 pacientes con obesidad grado III (16\%). En el Gráfico 8, se observa que los hombres presentan un mayor número con sobrepeso y obesidad grado I que otros grados de obesidad.
En el Gráfico 9, con datos de ambos sexos, se constata que la mayoría, $74 \%$ de los pacientes tienen enfermedades asociadas al sobrepeso u obesidad, siendo las enfermedades cardiovasculares con $72 \%$ las que más afectan a los pacientes con exceso de peso. Con menor porcentaje (1\%), se incluye la diabetes mellitus 2 y un caso específico de artrosis y cardiovascular, representando los casos en que hay dos enfermedades asociadas a obesidad. Por último, el $26 \%$ que no tuvieron enfermedad asociada diagnosticada. 


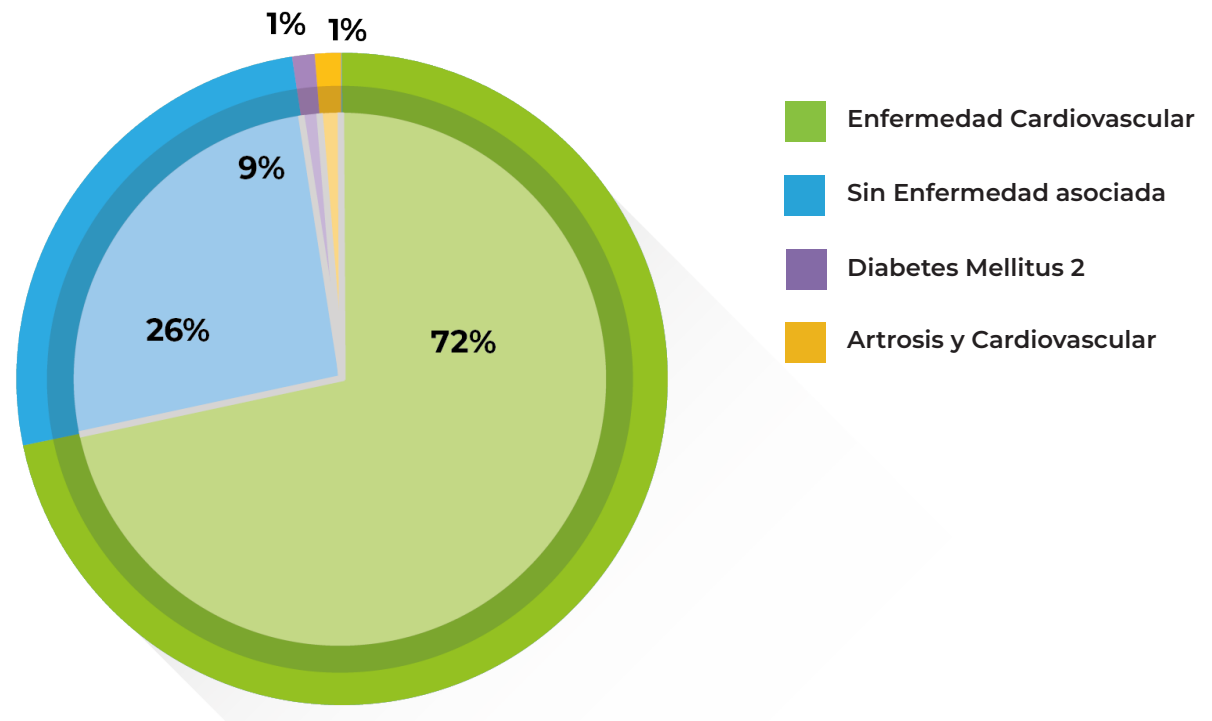

Gráfico 9. Porcentaje de pacientes de ambos géneros por enfermedad asociada al sobrepeso u obesidad.

En los gráficos 10 y 11 se exponen los porcentajes de pacientes con sobrepeso $\mathrm{u}$ obesos, que poseen enfermedades asociadas al exceso de peso, por separado, por género. De este modo, podemos hacer un comparativo entre el porcentaje de pacientes hombres y mujeres con enfermedades asociadas, analizando si las enfermedades

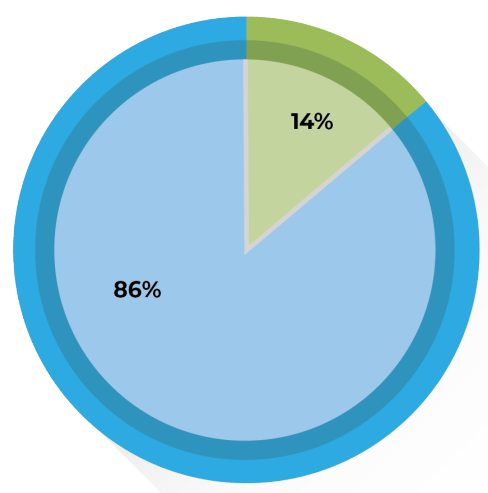

Enfermedad Cardiovascular

Sin enfermedad asociada

Gráfico 10. Pacientes hombres con enfermedades asociadas a sobrepeso y obesidad. asociadas al exceso de peso afectan igualmente ambos sexos o si hay algún género que sufre más impacto con estas enfermedades asociadas que son, en su gran mayoría las enfermedades cardiovasculares, conforme vimos en el gráfico con los dos géneros juntos.
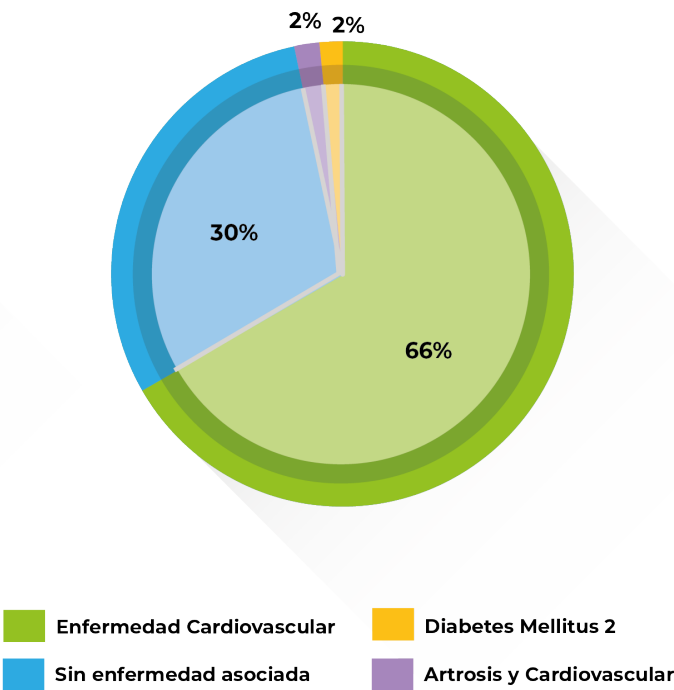

Gráfico 11. Pacientes mujeres con enfermedades asociadas a sobrepeso y obesidad. 
En el Gráfico 10 se puede observar que $86 \%$ de ellos sufre de enfermedad cardiovascular asociada al exceso de peso, mientras que $14 \%$ no poseen ninguna enfermedad asociada diagnosticada. Al hacer un revisión de los gráficos anteriores relacionados al grado de obesidad en que se encontraba cada género, los datos de los pacientes hombres poseían, $90 \%$ presentaban sobrepeso u obesidad grado I.

En el Gráfico 11, de pacientes mujeres, se puede destacar una mayor diversidad de enfermedades asociadas al exceso de peso,
$66 \%$ de pacientes mujeres con enfermedades cardiovasculares, $2 \%$ diabetes mellitus 2 y $2 \%$ artrosis y cardiovascular. Sin embargo, si se suman todas las enfermedades $(70 \%)$, los porcentajes no alcanzan el porcentaje masculino (86\%). El porcentaje de pacientes mujeres que no presentan enfermedades asociadas es de $30 \%$, esto es un poco más del doble en comparación con los mismos porcentajes que se muestran en el Gráfico sobre pacientes masculinos sin enfermedades asociadas (14\%).

\section{DISCUSIÓN}

La etiopatogenia comprende que la obesidad es la acumulación excesiva de grasa como resultado del balance energético positivo determinado por alteraciones en la ingesta y/o el gasto. Este balance energético es controlado por la hormona Leptina, producida en el tejido adiposo, que actúa como mensajero al sistema nervioso central (SNC) de las reservas energéticas almacenadas en el tejido adiposo. Cuando la cantidad de energía es insuficiente, las concentraciones de Leptina disminuyen y se activan los mecanismos de ingesta y ahorro energético, mediante la disminución de la termogénesis e inactivación de los sistemas prescindibles como el reproductor e inmunitario (4).

La Obesidad se presenta cuando el consumo de energía (Kcal) es mayor al que utiliza en el organismo, lo que origina aumento de peso corporal (5). Las necesidades de energía se basan por la tasa metabólica (o metabolismo) basal, que corresponde al gasto energético mínimo para la supervivencia. Una persona, aunque permanezca en reposo completo, consume mucha energía para todas las reacciones químicas del organismo (6).

La necesidad calórica diaria de las muje- res es menor que la que el hombre necesita, también es posible verificar que, en ambos sexos, las necesidades calóricas diarias van disminuyendo conforme la ancianidad se aproxima. Otro punto importante es que independiente de sexo, una persona sedentaria, necesita de bien menos calorías diarias do que personas que practican actividades físicas diarias como, por ejemplo, los trabajadores manuales y atletas.

El alto consumo de alimentos industrializados, que cada día sustituyen en mayor medida a los preparados en casa, el acelerado ritmo de vida de las ciudades, los tiempos prolongados de traslados al hogar, así como la mayor incorporación de la mujer al medio laboral favorecen la aceptación de productos conocidos como fast food a la dieta familiar, que propician el consumo de un alto porcentaje de grasa (5).

En la primera encuesta nacional de factores de riesgo de enfermedades no transmisibles realizada durante el año 2011, que tuvo como muestra, 2.538 paraguayos/as de entre 15 a 74 años. Los datos estadísticos de esta encuesta revelaron que 74,5\% de los paraguayos presentaron actividad física escasa o baja en el tiempo libre, es 
decir, son personas sedentarias y con alto riesgo de contraer enfermedades como la obesidad, diabetes, hipertensión arterial o problemas cardiovasculares (7).

\section{CONCLUSIONES}

La mayoría de los pacientes que buscan ayuda médica nutricional son en gran mayoría obesos, teniendo en cuenta el soma de los que tienen grado de obesidad I, II y III, equivalente al $62 \%$ de los pacientes y el 38\% restante son pacientes con sobrepeso diagnosticado. Los resultados determinaron 30 pacientes con sobrepeso (38\%), 27 pacientes con obesidad grado I (35\%), 12 pacientes con obesidad grado II (15\%) y 9 pacientes con obesidad grado III (12\%). En el grado de obesidad por
El sobrepeso u obesidad constituye un factor de riesgo en términos epidemiológicos, una persona con sobrepeso u obesa tiene mayores probabilidades de desarrollar otras enfermedades asociadas.

género, poco más de $90 \%$ de los pacientes masculinos eran hombres con sobrepeso y con obesidad grado I. En los resultados de pacientes femeninos, $16 \%$ tienen obesidad grado III y $17 \%$ obesidad grado II, un tercio de las mujeres con grado más severo de obesidad. El 74\% de los pacientes tienen enfermedades asociadas, siendo las enfermedades cardiovasculares con $72 \%$, las que más afectan a los pacientes con exceso de peso.

\section{REFERENCIAS}

1. OMS, Nota Descriptiva sobre la Obesidad y el Sobrepeso, 2018, disponible en: https://www.who.int/es/news-room/ fact-sheets/detail/obesity-and-overweight . Acceso en 28 octubre 2019.

2. Dirección General de Estadísticas, Encuestas y Censos. Proyecciones de población nacional, áreas urbana y rural, por sexo y edad. 2019. Disponible en: https:// www.dgeec.gov.py/Publicaciones/Biblioteca/proyeccion\%20nacional/Paraguay_ Triptico_2019.pdf Acceso en 26 noviembre 2019.

3. Ministerio de Salud Pública y Bienestar Social del Paraguay. Estrategia Nacional para la Prevención y el Control de la Obesidad 2015-2025. Asunción, Paraguay, 2015.
4. Vidal-Puig, A. Obesidad y Síndrome Metabólico. Capítulo 224. Medicina Interna. 18a Ed. Volumen 2. España: Editorial Elsevier España, 2016.

5. Esquivel Hernández, R. / Martínez Correa J. / Martínez Correa S. Nutrición y Salud. 3a Ed. México: Editorial El Manual Moderno, 2014.

6. Guyton y Hall. Tratado de Fisiología Médica. Capítulo 73. 13a Ed. España: Editorial Elsevier España, 2017.

7. Ministerio de Salud Pública y Bienestar Social del Paraguay. Primera Encuesta Nacional de Factores de Riesgo de Enfermedades No Transmisibles. 1a Ed. Asunción, Paraguay, 2012. Disponible en: http://portal.mspbs.gov.py/dvent/wp-content/uploads/2015/10/Encuesta-Nacional. pdf . Acceso en 24 noviembre 2019. 